\title{
A Population-Based Seroprevalence Survey of Severe Acute Respiratory Syndrome Coronavirus 2 Infection in Beijing, China
}

\section{Xiaoli Wang}

Beijing Center for disease prevention and control

\section{Wenjing Gao}

Peking University School of Public Health

\section{Shujuan Cui}

Beijing Centers for Disease prevention and control

\section{Yi Zhang}

Beijing center for disease prevention and control

\section{Ke Zheng}

Peking University School of Public Health

Ji Ke

Peking University School of Public Health

Jun Lv

Peking University School of Public Health

\section{Canqing Yu}

Peking University School of Public Health

\section{Dianjianyi Sun}

Peking University School of Public Health

Quanyi Wang ( $\square$ bjcdcxm@126.com )

Beijing Center for Disease Prevention and Control

\section{Liming Li}

Peking University School of Public Health

\section{Research article}

Keywords: COVID-19, SARS-CoV-2, asymptomatic case, serological survey

Posted Date: January 8th, 2021

DOl: https://doi.org/10.21203/rs.3.rs-141246/v1 
License: (c) (i) This work is licensed under a Creative Commons Attribution 4.0 International License. Read Full License 


\section{Abstract}

\section{BACKGOUND}

The spread of Coronavirus Disease 2019 (COVID-19) had been substantially controlled in China. As the capital of China, Beijing took a series of strict containment measures during the past several months. However, the seroprevalence of COVID-19 in Beijing has not been evaluated.

\section{METHODS}

During April 15-18, 2020, residents in Beijing were enrolled using a multi-stage cluster random sampling technique in four districts with top COVID-19 incidence and one district without case reported. Communities and households in each district were randomly selected based on probability proportional to size. Blood samples were collected and antibodies to severe acute respiratory syndrome coronavirus 2 (SARS-CoV-2) were tested by two kinds of colloidal gold kits. All colloidal gold positive serums and 20 randomly selected negative serums were then tested by Micro-neutralization assay. A likelihood ratio test was used to estimate $95 \%$ confidence intervals of seroprevalence with the Clopper-Pearson exact method.

\section{RESULTS}

A total of 2,184 residents participated in this survey, among which 13 were tested positive by colloidal gold tests. Of those who tested positive, 8 were IgM positive, 3 were IgG positive, 1 was total antibody positive, 1 was both IgM and total antibody positive. Among the 13 seropositive samples and 20 randomly selected seronegative samples, no positive sample was detected by micro-neutralization assay. The seroprevalence of COVID-19 in Beijing was estimated no higher than $0.17 \%$.

\section{CONCLUSIONS}

The seroprevalence of COVID-19 was low in April suggests that the comprehensive control measures to prevent and control further spread in Beijing was effective. However, the majority of the residents in Beijing were still susceptible to infection; the risk of rebound should be noted due to low population-level immunity.

\section{Background}

Coronavirus Disease 2019 (COVID-19) has rapidly spread throughout the world. Reported cases only represented a small fraction of the real number of cases. According to a few early studies from Iceland, US and Iran, the seropositivity at community level ranged from $0.6 \%-22 \%$, much higher than the level of reported incidence rate [1-4]. Investigating seroprevalence in different locations around the world enables further understanding the infection spectrum, the incidence and case-fatality rate, and population immunity level of severe acute respiratory syndrome coronavirus 2 (SARS-CoV-2), and optimizing strategies for disease prevention and control. 
COVID-19 was initially reported in Wuhan, China. With the rapid spread of SARS-CoV-2 all around the world, Beijing - the political, cultural and international exchange center of China- faced an extraordinary challenge both at home and abroad. The first COVID-19 case in Beijing was confirmed on January 19th, 2020. The authority in Beijing implemented a series control measures to prevent further spread during the past several months. As of April 15, a total of 594 COVID-19 cases including 9 deaths were officially confirmed and reported in Beijing.

To evaluate the effectiveness of control strategies and the risk of future epidemic in Beijing, a community-based serological survey was performed.

\section{Method}

\section{Study design}

A community-based, age stratified, onetime cross-sectional serological survey in 2,184 adults and children from 25 communities were conducted in Beijing. The participants were investigated during April 15 to 18. This study had been approved by the IRB at Peking University Health Science Center prior to participant enrollment (IRB00001052-20021). Written informed consents were acquired from all participants in the investigation. For child $<18 \mathrm{yrs}$, consent had been obtained from his/her legal guardian.

\section{Study population}

Residents who aged $>1$-year-old, lived in Beijing for at least 14 days in between of January and March 2020 were eligible for participation.

\section{Sampling strategies}

This survey was performed by a multi-stage cluster random sampling technique. The highest four incidence districts (Xicheng, Shijingshan, Daxing and Fengtai) and the lowest incidence district (Pinggu with no confirmed case reported) out of 16 districts in Beijing were selected according to the protocol by World Health Organization [5]. Five communities in each district and certain households in each community were randomly selected based on Probability Proportionate to Size (PPS) at two stages respectively. A household was defined as a group of people (1 or more) living in the same residence. All persons living in the household had been invited to participate in the study, including children. If the age structure of samples were not consistent with that of general population in targeted communities due to refusal or contraindication to venipuncture, additional households were randomly selected to ensure the representativeness of samples.

\section{Data collection}

We recruited participants organized by community residential committee. Each participant enrolled in survey was investigated using a questionnaire which covers demographic information and COVID-19 relevant exposure information. 


\section{Specimen collection}

A certain amount of whole blood sample was drawn into vacutainer without anticoagulant from each participant ( $5 \mathrm{ml}$ for $5+$ years old, $3 \mathrm{ml}$ for the rest) and transported to the local Center for Disease Prevention and Control (CDC) laboratory. The serum was separated and apportioned into two aliquots $A$ and $B$. The serum in tube $A$ was transported to Beijing CDC laboratory for SARS-CoV-2 antibody testing, while tube B was stored backup.

\section{Serological testing}

Serum samples were screened for the presence of SARS-CoV-2 specific antibodies using two different antibody test (Colloidal Gold) kits, Wondfo (Guangzhou, Batch W19500315) and Innovita (Tangshan, Batch 20200402), which had been approved by China Food and Drug Administration (CFDA). According to the manufacturer, test performance characteristics showed a sensitivity of $₫ 86.4 \%$ and a specificity of ه99.6\%.

If a serum sample was positive when using either test kit, the serum in tube B (backup serum) would be couriered to China CDC for a micro-neutralization assay to measure and confirm the SARS-CoV-2 -specific neutralizing antibody. For seronegativity in colloidal gold tests, 20 random samples were confirmed. The diluted serums were mixed with a virus suspension of 100 TCID50 (50 tissue culture infective dose) in 96well plates at a ratio of 1:1. Cytopathic effect (CPE) of each well was recorded under microscopes, and the neutralizing titer was calculated by the dilution number of $50 \%$ protective condition. A titer of $1: 4$ or higher indicated seropositivity.

\section{Statistical analyses}

Age-specific cumulative incidence was the proportion of participants per age strata who tested seropositive for SARS-CoV-2 infection. Proportion were adjusted for difference in the age structure of the participants and the overall population. Population weighting estimation and more details were shown in the Statistical Appendix.

We used a likelihood ratio test to calculate $95 \%$ confidence intervals of fractions with the Clopper-Pearson exact method (when the estimated fraction was 0), as implemented in the R package binom [3].

\section{Results}

From 39,769 households in 5 districts of Beijing, 578 households, including 1,510 subjects in the sampling protocol participated in this study. Considering the age structure of 1,510 subjects were not consistent with that of the local populations, additional subjects were randomly selected in certain age groups. Between April 15 and 18, 2020, a total of 1,247 households including 2,184 participants were enrolled in this research.

Table 1 provided demographic characteristic of unadjusted sample and population-weight adjusted of the sample and Beijing estimates. The sample distribution was not significantly deviated from that of 
Beijing.

Table 1

Demographic characteristics of 2,184 study participants

\begin{tabular}{|lll|}
\hline Characteristics & $\mathbf{N}(\%)$ & Adjusted proportion (\%)* \\
\hline Sex & & \\
\hline Men & $997(45.7)$ & $51.6(45.7)$ \\
\hline Women & $1187(54.3)$ & 48.4 \\
\hline Age group & & \\
\hline $1-9$ & $172(7.9)$ & 6.1 \\
\hline $10-19$ & $164(7.5)$ & 8.0 \\
\hline $20-39$ & $643(29.4)$ & 43.6 \\
\hline $40-59$ & $714(32.7)$ & 29.8 \\
\hline$\geq 60$ & $491(22.5)$ & 12.5 \\
\hline Occupation & & \\
\hline Child/Student & $355(16.3)$ & 15.2 \\
Farmer & $211(9.7)$ & 3.9 \\
Worker & $182(8.3)$ & 9.5 \\
Officer & $136(6.2)$ & 6.1 \\
Doctor/Nurse & $42(1.9)$ & 2.3 \\
Teacher & $19(0.9)$ & 1.1 \\
Retiree & $518(23.7)$ & 16.1 \\
Service Worker & $172(7.9)$ & 13.1 \\
Delivery Staff & $5(0.2)$ & 0.6 \\
Policeman/Security & $20(0.9)$ & 1.5 \\
Taxi driver & $15(0.7)$ & 0.7 \\
Housekeeper & $24(1.1)$ & 1.2 \\
Community staff & $145(6.6)$ & 9.4 \\
Other & $340(15.6)$ & 19.3 \\
\hline
\end{tabular}


Thirteen of the 2,184 serum samples were tested seropositive by Colloidal Gold tests, among which 8 were IgM positive, 3 were IgG positive, 1 was total antibody positive, 1 was both IgM and total antibody positive. None of the 13 colloidal gold positive individuals reported any possible exposure (Table 2).

Table 2

Serological results in 2,184 study participants.

\begin{tabular}{|c|c|c|c|}
\hline Variable & $\begin{array}{l}\text { Total }(\mathrm{N} \\
=2184)\end{array}$ & $\begin{array}{l}\text { Colloidal gold test } \\
\text { Positive }(\mathrm{N}=13)\end{array}$ & $\begin{array}{l}\text { Micro-neutralization assay } \\
\text { Positive }(\mathrm{N}=0)\end{array}$ \\
\hline Male sex - no. (\%) & $997(45.7)$ & $7(53.9)$ & - \\
\hline Mean age - yr & $\begin{array}{l}42.3 \pm \\
19.5\end{array}$ & $45.6 \pm 18.3$ & - \\
\hline \multicolumn{4}{|l|}{ Diseases - no. (\%) } \\
\hline $\begin{array}{l}\text { Pregnant } \\
\text { Women - no. (\%) }\end{array}$ & $4(0.2)$ & $0(0)$ & \\
\hline Any travel - no. (\%) & $63(2.9)$ & $0(0)$ & - \\
\hline \multicolumn{4}{|l|}{ Specific travel - no. (\%) } \\
\hline Wuhan & $3(0.1)$ & $0(0)$ & - \\
\hline $\begin{array}{l}\text { Hubei Province* (Wuhan } \\
\text { excluded) }\end{array}$ & $46(2.1)$ & $0(0)$ & - \\
\hline International travel & $15(0.7)$ & $0(0)$ & - \\
\hline $\begin{array}{l}\text { Contact of patients with } \\
\text { symptom(s)**-no. (\%) }\end{array}$ & $29(1.3)$ & $0(0)$ & - \\
\hline $\begin{array}{l}\text { Contact with infected cases - } \\
\text { no. }(\%)\end{array}$ & $3(0.1)$ & $0(0)$ & - \\
\hline $\begin{array}{l}\text { Reported symptom(s) })^{\star \star}-\text { no. } \\
(\%)\end{array}$ & $75(3.4)$ & $0(0)$ & - \\
\hline $\begin{array}{l}\text { Reported any clinic visit } \\
\text { history - no. (\%) }\end{array}$ & $53(2.4)$ & $0(0)$ & - \\
\hline $\begin{array}{l}\text { Reported being diagnosed of } \\
\text { COVID-19 }\end{array}$ & $0(0)$ & $0(0)$ & - \\
\hline \multicolumn{4}{|c|}{ * Wuhan is the capital city of Hubei province. } \\
\hline $\begin{array}{l}\text { ** All-caused respiratory symp } \\
\text { limit to COVID-19. }\end{array}$ & includin & ver, cough, shortr & reath, or pneumonia, do not \\
\hline
\end{tabular}

Among 13 seropositive samples and 20 randomly selected seronegative samples, none was positive in neutralization assay. 
Considering no positive result in neutralization assay, we used a likelihood ratio method to calculate $95 \%$ confidence intervals of fractions with the Clopper-Pearson exact method. The seroprevalence of COVID19 was estimated not higher than $0.17 \%$ with $95 \%$ confidence in Beijing.

\section{Discussion}

In this study, no positive neutralizing antibodies case was detected by micro-neutralization assay. We estimated that the percentage of participants that tested positive in population screening were not higher than $0.17 \%$ with $95 \%$ confidence during April 15-18, which indicates Beijing's control effort had stopped community transmission of SARS-CoV-2 substantially. Meanwhile, these results also showed that the majority of populations in Beijing remained susceptible to SARS-CoV-2. Indeed, Beijing experienced a COVID-19 rebound in June. On June 11, a new COVID-19 case was reported in Beijing after no new confirmed infection for 56 consecutive days.

To date, micro-neutralization assay, as the gold-standard is the most specific and sensitive serological assay for evaluating and detecting functional neutralizing antibodies [6]. However, the neutralizing antibodies of asymptomatic cases decreased within 2-3 months after infection [7]. Therefore, the seroprevalence might be underestimated if: (1) the serum sample was not collected within the window of neutralizing antibody production; (2) the immune response of asymptomatic infection was not strong enough to be detected.

Several research teams worldwide had started testing samples for SARS-CoV-2 antibodies. Among high risk populations, the average seroprevalence in healthcare a tertiary hospital in Germany in employees of a university hospital emergency department in USA was 1.6\% [8] and 5.9\% [9] respectively. Among blood donors, 4.4-10.8\% in Milan of Italy [10] and 5/500 in Scotland of UK [11] were positive for anti- SARSCoV-2. Among general populations, $0.6 \%$ [3], 1.5\% [2], 4.1\% [4] and 22\% [1] were antibody positive in random community participants in four surveys conducted in Iceland, Santa Clara and Los Angeles County of USA and Guilan of Iran respectively. The varying rates of COVID-19 antibody seropositivity in above studies emphasized the importance of serological survey in different populations. More studies were needed to determine the seroprevalence in community residents in both high and low COVID-19 epidemic regions of China.

Our study has several limitations. One of the main limitations is the performance of test kits. According to the manufacturer, the two test kits were both used for the additional testing for suspected case with negative PCR, which indicated that they are unsuitable for general population screening. Although none was positive in neutralization assay among these 13 seropositive samples and 20 randomly selected seronegative samples, we could not preclude the possibility of false negativity since the neutralization assay was conducted only in targeted part of residents. Despite the acceptable sensitivity of the two colloidal gold methods provided by the manufacturers, Döhla et al. found that antibody-based rapid test showed low sensitivity (36.4\%) in high-prevalence community setting. They recommended not to rely on an antibody-based rapid test for public health measures such as community screenings [12]. Additional 
validation of the assays especially in general populations used could improve our estimates. In addition, inadequate sample size and one-time cross-sectional study performed were also needed to be considered. Given the low seroprevalence in Beijing, larger sample size is expected to be further investigated in order to achieve dynamic and more accurate seroprevalence estimation.

\section{Conclusion}

The seroprevalence of COVID-19 was low in Beijing by April, which suggests that the comprehensive control measures effectively prevent and contain further spread in Beijing. However, the majority of the residents in Beijing were susceptible to infection and the risk of rebound should be noted due to the low population-level immunity.

\section{Abbreviations}

COVID-19: coronavirus; Coronavirus Disease 2019; SARS-CoV-2: Severe Acute Respiratory Syndrome Coronavirus; PPS: Probability Proportionate to Size; CDC: Center of Disease Prevention and Control; CFDA: China Food and Drug Administration; CPE: Cytopathic effect.

\section{Declarations}

\section{-Ethics approval and consent to participate}

This study had been approved by the IRB at Peking University Health Science Center prior to participant enrollment (IRB00001052-20021). Written informed consents were acquired from all participants in the investigation.

\section{-Consent for publication}

Not applicable.

\section{-Availability of data and materials}

The datasets used and/or analysed during the current study are available from the corresponding author on reasonable request.

\section{-Competing Interests}

The authors declare that there is no conflict of interests.

\section{-Authors Contributions}

Concept and design: LL and QW.

Acquisition, analysis, or interpretation of data: All Authors. 
Drafting of the manuscript: XW and WG.

Critical revision of the manuscript: LL and QW.

Laboratory testing: SC.

Data management and statistical analysis: $\mathrm{XW}, \mathrm{WG}, \mathrm{YZ}, \mathrm{KZ}, \mathrm{JK}, \mathrm{JL}, \mathrm{CY}$, and DS

\section{Acknowledgement}

We thank China CDC for assistance with study design and laboratory support. The authors would like to thank all the investigators from Xicheng, Shijingshan, Daxing, Fengtai, and Pinggu CDC for field investigation and data collection and all participants for their contribution in this study.

\section{Funding/Support}

The study was supported by the National Natural Science Foundation of China (82041027), Special Research Fund of PKU for Prevention and Control of COVID-19 and the Fundamental Research Funds for the Central Universities. The funders had no role in the study design, data collection and analysis, decision to publish, or preparation of the manuscript.

\section{References}

1. Shakiba M, Nazari SSH, Mehrabian F, et al. Seroprevalence of COVID-19 virus infection in Guilan province, Iran. https://www.medrxiv.org/content/10.1101/2020.04.26.20079244v1.

2. Bendavid E, Mulaney B, Sood N, et al. COVID-19 Antibody Seroprevalence in Santa Clara County, California. https://www.medrxiv.org/content/10.1101/2020.04.14.20062463v2.

3. Gudbjartsson DF, Helgason A, Jonsson H, et al. Spread of SARS-CoV-2 in the Icelandic Population. N Engl J Med. 2020;382:2302-15.

4. Sood N, Simon P, Ebner P, et al. Seroprevalence of SARS-CoV-2-Specific Antibodies Among Adults in Los Angeles County, California, on April 10-11, 2020. JAMA 2020; 323(23):2425-7.

5. World Health Organization. Population-based age-stratified seroepidemiological investigation protocol for COVID-19 virus infection. https://www.who.int/publications/i/item/WHO-2019-nCoVSeroepidemiology-2020.2. Accessed 14 September 2020.

6. Manenti A, Maggetti M, Casa E, et al. Evaluation of SARS-CoV-2 neutralizing antibodies using of a CPE-based Colorimetric live virus micro-neutralization assay in human serum samples. J Med Virol, 2020; [Epub ahead of print].

7. Long QX, Tang XJ, Shi QL, et al. Clinical and immunological assessment of asymptomatic SARSCoV-2 infections. NAT MED. 2020;26:1200-4. 
8. Korth J, Wilde B, Dolff S, et al. SARS-CoV-2-specific antibody detection in healthcare workers in Germany with direct contact to COVID-19 patients. J CLIN VIROL 2020; [Epub ahead of print].

9. Madsen T, Levin N, Niehus K. Prevalence of IgG antibodies to SARS-CoV-2 among emergency department employees. AM J EMERG MED. 2020;S0735-6757(20):30306-5.

10. Valenti L, Bergna A, Pelusi S, et al. SARS-CoV-2 seroprevalence trends in healthy blood donors during the COVID-19 Milan outbreak. https://www.medrxiv.org/content/10.1101/2020.05.11.20098442v2..

11. Neutralising antibodies to SARS coronavirus 2 in Scottish blood donors - a pilot study of the value of serology to determine population exposure.

https://www.medrxiv.org/content/10.1101/2020.04.13.20060467v2..

12. Dohla M, Boesecke $C$, Schulte $B$, et al. Rapid point-of-care testing for SARS-CoV-2 in a community screening setting shows low sensitivity. PUBLIC HEALTH. 2020;182:170-2.

\section{Supplementary Files}

This is a list of supplementary files associated with this preprint. Click to download.

- 20200523StatisticalAppendix11.docx 